\title{
An Approach to Safety Analysis of Clinical Workflows
}

\author{
By Lamis Al-Qora'n* \\ Neil Gordon ${ }^{*}$ \\ Septavera Sharviat \\ Martin Walker ${ }^{+}$ \\ Yiannis Papadopoulos
}

\begin{abstract}
A clinical workflow considers the information and processes that are involved in providing a clinical service. They are safety critical since even minor faults have the potential to propagate and consequently cause harm to a patient, or even for a patient's life to be lost. Experiencing these kinds of failures has a destructive impact on all the involved parties. Due to the large number of processes and tasks included in the delivery of a clinical service, it can be difficult to determine the individuals or the processes that are responsible for adverse events, since such an analysis is typically complex and slow to do manually. Using automated tools to carry out an analysis can help in determining the root causes of potential adverse events and consequently help in avoiding preventable errors through either the alteration of existing workflows, or the design of a new workflow.

This paper describes a technical approach to safety analysis of clinical workflows, utilising a safety analysis tool (HierarchicallyPerformed Hazard Origin and Propagation Studies (HiP-HOPS)) that is already in use in the field of mechanical systems. The paper then demonstrates the applicability of the approach to clinical workflows by applying it to analyse the workflow in a radiology department. We conclude that the approach is applicable to this area of healthcare and provides a mechanism both for the systematic identification of adverse events and for the introduction of possible safeguards in clinical workflows.
\end{abstract}

\section{Introduction}

The increasing use of information systems in delivering clinical processes brings about substantial benefits to healthcare industry. At the same time, these

\footnotetext{
*PhD Student, University of Hull, UK.

${ }^{\dagger}$ Senior Lecturer, University of Hull, UK.

${ }^{\ddagger}$ Research Assistant, University of Hull, UK.

${ }^{+}$Lecturer, University of Hull, UK.

'Professor, University of Hull, UK.
} 
innovations require changes to established processes and can create complex workflows which have the potential to affect patient safety by causing unintended faults within clinical services, which can be harmful to individuals and may affect the reputation of healthcare organizations. Therefore, in order to reduce this risk and to achieve benefits from the involvement of advanced applications and processes, attention should be given to the design and the safety of clinical workflows. A workflow defines the activities planned to accomplish a process, the order of invocation of activities, the conditions under which such activities must be invoked, and the synchronization and information flow (dataflow) between activities (Belhajjame et al., 2001). Business process re-engineering used the term workflow to refer to the processes involved in arriving at a given objective which can be completed only through certain steps, and handled by more than one person (Ouvry, 2002). So, workflow can be understood as the description of a process model, and management of automated and manual tasks of business rules (Fischer, 2001).

Healthcare processes have both medical and non-medical activities which can be considered within a particular workflow; both types include potentially high-risk activities and failure in any part may lead to catastrophic consequences (Ruffolo et al., 2007). In other words, a healthcare workflow includes medical and administrative activities that are executed to produce a clinical service; deviations in these activities have the potential to deliver a faulty service.

Referring to the previous definitions, we define the term "clinical workflow" to describe the tasks that are performed carefully by more than one participant to accomplish a clinical process (e.g. treatment or diagnosis) and to produce a certain clinical output.

It has been widely reported that different healthcare errors, including those in medical treatment processes and workflows, are significant cause of death and suffering (e.g. see (Starfield, 2000) and (Green, 2013)). As patient safety has come to be an international priority (Battles and Lilford, 2003), finding effective methods to reduce medical errors and their contribution to adverse events is required (Murff et al., 2003).

In clinical workflows, we may face the problem of having errors in the developed workflow (which is supposed to be followed by the involved parties) that may propagate to cause failures and output deviations. We can avoid having such kind of errors by applying a formal systematic method for the development and the safety analysis of the workflow. Human failures are also expected in any large process, so a system is susceptible to this hazard if there is no technological remedy (Reason, 1990). In this research, human errors are to be considered through the workflow safety analysis because this analysis focuses on the errors and faults in the workflows which happen during executing the steps of the workflow and leads to workflow failures.

Due to the large number of parties typically involved in the delivery of clinical service, it can be difficult to determine the individuals or the processes which are responsible for the adverse events. Using technological tools to do so 
can help in determining the root causes of the potential adverse events. This enables the redesign of the processes and tasks which are accountable for such events, and consequently improves the reliability of the workflow through designing more reliable and non-faulty workflows. The analysis results can help as well to improve training of medical staff.

During the workflow development and safety analysis, all possible failure modes should be considered. Safe operation of a workflow in action is not guaranteed unless the theoretical developed workflow is carefully checked for safety and followed exactingly by the involved agents. Currently used methodologies, such as manual Fault Tree Analysis (FTA) and Failure Mode and Effect Analysis (FMEA) rely on the ability of the safety engineer to understand and to predict the workflow behaviour. So, they themselves are difficult to validate for human error. Automation of these methods can help when dealing with more complex workflows.

This paper presents a systematic technical approach to safety analysis of clinical workflows. The focus of the approach is on the integration between the architectural design of the systems and applications (that are part of the workflow), the dataflow within the involved systems, and the system environment: in other words, the interactions between the different parties involved in the clinical workflow. These may include the physicians, radiologists, nurses, patients, and the system environment, and all must be modelled and analysed in combination with the hardware and software components.

Modelling these interactions and focusing on the relations and the communication between all agents for the purpose of the safety analysis can both achieve a more holistic analysis and provide an efficient way to identify preventable errors and solutions for potential failures, and therefore helps in redesigning the workflow to achieve best safety results and accordingly to provide safe healthcare practices.

We will demonstrate the development and safety analysis of a workflow as a structured approach through which safety analysis tools are used to reduce risk over the workflow. In other words, the approach should increase the reliability of workflows through the safety analysis. We define a workflow as safe once it can be executed without failures, if the detailed steps in the workflow are executed correctly. It is crucial that comprehensive approaches be developed so that different components and properties of the clinical workflow can be analysed. The analysis of a clinical workflow.

This paper is structured as follows. In the next section we present our proposed approach, where the requirement gathering and workflow modelling are discussed. A technique called Hierarchically-Performed Hazard Origin and Propagation studies (HiP-HOPS) is used to automate the FTA and FMEA of the workflow. Probability analysis is optional, and the new workflow design and accreditation is also presented. In section four, we illustrate the approach through an example of an analysis of a workflow in a radiology department. Finally, we draw our conclusions and discuss related work. 


\section{An Approach for Safety Analysis of Clinical Workflows}

The focus of the workflow safety analysis must be on analysing the safety requirements in the presence of component failures (components here are hardware, software, humans, processes and tasks). The use of Model Based Safety Analysis (MBSA) is recommended, where models for the workflow are developed and repeated analysis and redevelopment on the model is performed until an accreditable workflow design is achieved. The model can be developed by the safety engineer in cooperation with a group of healthcare experts. After the model is developed the modelling notation itself must be annotated with the fault behaviour. A graphical language such as Simulink can be used for modelling.

The proposed approach aims is designed to support the development and safety analysis of clinical workflows. The following daAiagram illustrates the approach:

Figure 1.The Approach

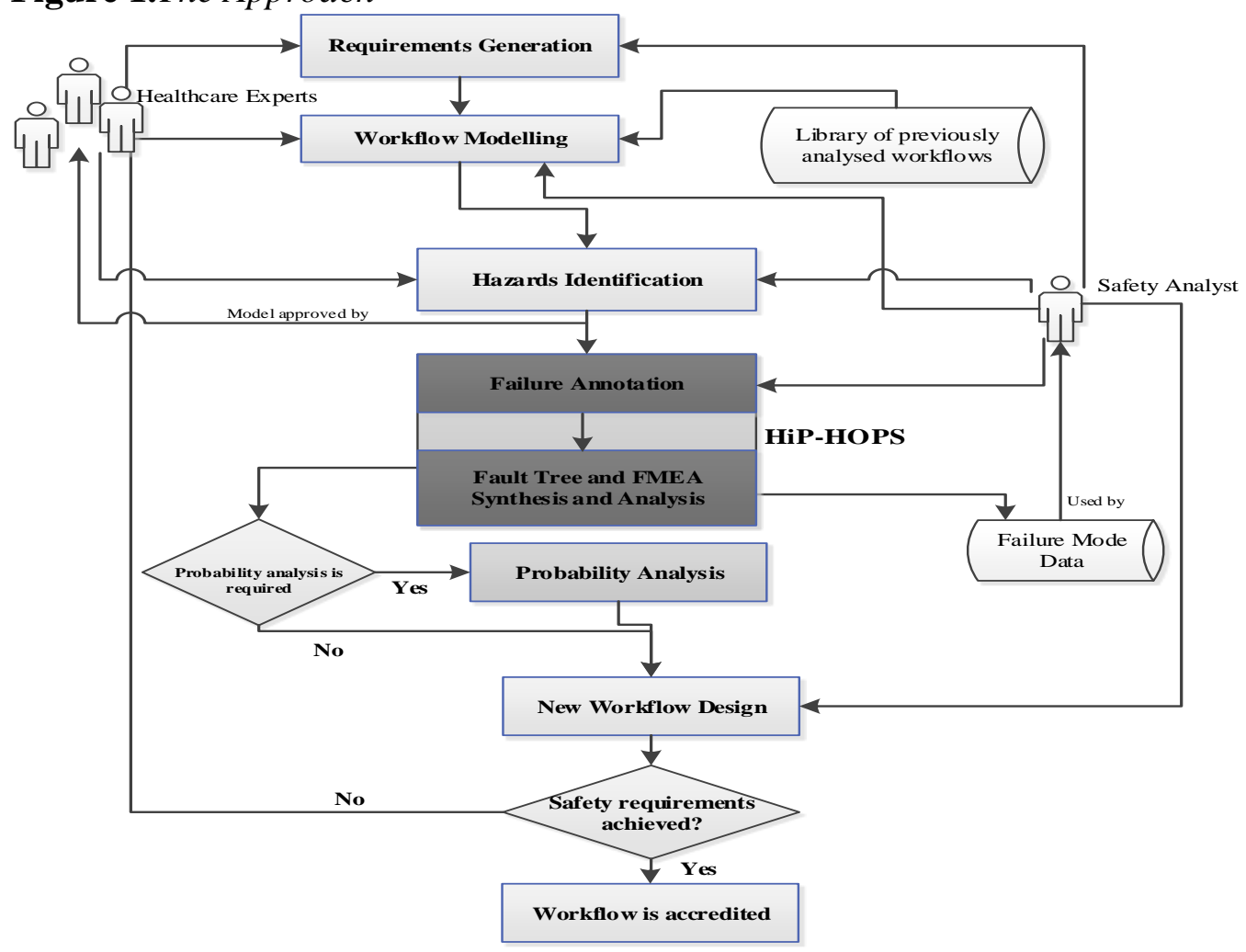

The following sections explain the approach in more detail.

Hierarchically-Performed Hazard Origin and Propagation Studies (HiPHOPS)

Hierarchically-Performed Hazard Origin and Propagation Studies (HiPHOPS) (Papadopoulos and McDermid, 1999) is a state of the art technique for identifying week points in systems design in an efficient manner. It allows 
semi-automation and integration of two important classical methods: FTA and FMEA.

FTA is a deductive method which describes how different components failures (or events) or their combinations can cause an unwanted event (often termed the top event). Quantitative analysis of the FTA can be implemented to calculate the probability of the top event and qualitative analysis is performed to identify the necessary and sufficient combinations of events which caused the top event (termed Minimal Cut Sets or 'MCS') (Vesely et al., 1981). FMEA is an inductive technique which inspects the effect of component failures towards system failures.

System models can be annotated with HiP-HOPS failure behaviour using a number of modelling packages (e.g. Matlab Simulink). These models are then analysed and FTA and FMEA results are produced.

Al-Qora'n et al. (2013) used HiP-HOPS to analyse the safety of the dataflow of a home Telemonitoring system. This current paper utilises HiPHOPS to develop an approach for safety analysis of clinical workflows. The approach considers the differences between healthcare and other industries as well as the differences between mechanical systems and clinical workflows.

\section{Phases of the Workflow Safety Process}

Requirements Generation

The first step in the safety analysis process is done by a safety engineer/analyst in the healthcare organization in cooperation with a team of healthcare experts. The safety engineer/analyst is responsible for collecting sufficient detailed data to model the workflow and its critical parts. In-depth data includes the involved parties, hardware and software components, processes and tasks involved in the workflow. The approach suggests that the safety engineer must collect the data about a new workflow from scratch instead of using any existing models for the purpose of the analysis. However, the approach proposes the use of previously analysed components in this phase and in the modelling; the decision to do this is done through this phase when the healthcare experts specify that the workflow could be connected with another workflow or parts of another workflow in the hospital. An individual workflow component can be a task, a process, a software agent, human agent, hardware, or a workflow that encapsulates a set of related tasks or processes.

\section{Workflow Modelling}

After amassing comprehensive data about the workflow, the available documented data needs to be organised and structured. The functional/ architectural model needs to be built, and it should show the basic topology of the workflow and the interconnections between various components and subcomponents. All workflow processes are placed into separate components so that all of the tasks and different agents inside each component are semantically related; that is why we can say that the components are integrated and interconnected. 
Hazards Identification

After building the model the possible ways the overall workflow can fail are to be determined. This is the responsibility of the safety engineer and the healthcare experts. They determine the possible faults in each component separately to provide sufficient data for the failure annotation.

\section{Failure Annotation}

After preparing the model, and specifying the failure behaviour for each of the component in the model, the process of annotating the model with the failure behaviour starts. The HiP-HOPS tool needs to know how the various components of the system are interconnected and how each can fail. In this phase components are annotated with a set of the failure expressions showing how deviations in each component output can be caused by either internal failure of this component or corresponding deviations in its input. In other words, the failure information defines how failure in the component output can be caused by the propagation of failure to the component input or the internal malfunction of the component itself.

So, each component (which could be a process, task, user, application, or hardware) needs to be annotated with its own failure data which describes what could go wrong with the component and how it responds to failures elsewhere in the system. For example, for an application component this data might include unexpected omission or unintended commission of the output.

\section{Fault Tree and FMEA Synthesis and Analysis}

After the model is annotated, HiP-HOPS is applied and automatically navigates through the failure information backwards, starting from the workflow failure and connecting each failure to its causes, linking them with logical operators. This results in the automated production of the main fault tree which can then be analysed. HiP-HOPS synthesises and analyses the workflow fault tree and produces the FTA and FMEA results which show how the value failure in components can lead to the workflow failure. During this phase, the MCSs that are required to cause the top event are obtained as well.

This qualitative analysis data are then used by the analyst to redesign the workflow. In some cases this qualitative data is enough to refine and reengineer the processes and tasks within the workflow. Therefore, the analyst can start to redesign the workflow immediately after the qualitative analysis. However, in some cases the new design may require replacements of the existing components with components which are costly for the healthcare organisation, and thus the healthcare providers may ask for quantitative information about the likelihood of the component to fail. In these cases, quantitative analysis is recommended, otherwise, the analyst can move immediately to the new workflow design phase.

\section{Probability Analysis}

Probability analysis is an optional step which can be carried out within the previous phases. This could be performed by obtaining the failure information 
of different components and adding numerical information into the components in the modelling phase, where HiP-HOPS provides information about the unavailability of each top event.

Workflow components could be processes, tasks, or people. Therefore, it is different from other components that have manufacturer failure rate. Human error failure rates can be acquired using quantitative analysis methods of real data to get statistical information from previous experiences. However, this method has the problem that the analysis results may reflect incorrect results due to the vagueness of the data provided. The reason behind this inaccuracy is that human failures are dynamic as they may be affected by many environmental factors. This was the motivation behind searching for another mechanism for the probability analysis which handles the uncertainty issue. Therefore, probability of the human error usually cannot be given an exact value, and instead would be estimated.

The proposed probability analysis in this approach is a single phase and is separated from the analysis phase. After finishing the analysis phase and getting the FTA and FMEA results, the analyst can start the quantitative analysis if it is required by getting an estimated probability for each of the fault tree components. In this case, analysts can seek experts' opinion and can develop their own tools to get such failure numeric information. After that, fuzzy set theory is used to quantify the fault trees.

This failure probability analysis approach has the potential to affect the results and the accuracy of the analysis. In other words, it specifies the failure rates that are used for the analysis and consequently has a direct effect on the analysis results.

\section{New Workflow Design}

After completing the analysis using HiP-HOPS and getting the qualitative data, the workflow needs to be redesigned and the workflow components, which have faults that might cause the failures, need to be replaced with components that are more reliable. As explained previously, if this replacement is costly for the hospital or the healthcare institution, they might require probability analysis for the workflow, as they want to see some statistics about the probability of those causes to make an informed decision about the cost of replacement. In other words, this is a part of the cost benefit analysis, where the cost of replacement is compared to its benefits and the severity of the failure.

If the probability analysis is required, it must be accomplished before the workflow redesign; if it is not required, workflow redesign is accomplished immediately after the qualitative analysis. After that, it needs to be tested if it meets the safety requirements or not; if yes, the safety engineer can go ahead and the workflow is accredited and approved as the formal workflow in the department. Conversely, if it not does meet the requirements, then the process needs to be repeated until the workflow is satisfactory.

When the safety engineer develops the workflow, the healthcare providers who are involved in the workflow and the various involved parties must be 
notified and safety training provided for them. This helps to ensure that they are aware of the causes and consequences of any potential errors within the workflow. The analysis results must be summarised as formal guidelines in a medical language instead of formal technical language. Every employee should have an understanding of how these results might show that certain failures are his/her responsibility.

\section{Accreditation of the Workflow}

Once the workflow is analysed and redesigned, and before the workflow can be accredited as the formal workflow within a department or a hospital, the safety engineer must make a thorough check to make sure that all the significant risks and hazards are addressed. Moreover, he should make sure that the remaining risk is acceptable and he should focus the attention of the users to this risk. Solutions for the possible failures must be effective. For example, a root cause for the failure which could be a certain task must be marked as it should be executed in a strict way.

One more important issue to keep in mind, when planning to adopt new systems or to make changes on the organisational procedures level, is that these changes must be reflected in the workflow itself. This should be done because these changes have the potential to introduce new hazards.

Using this approach has the potential to reveal patterns of diagnostic and procedural failures that suggest areas for improvement. Systematic analysis of such failures and pinpointing their root causes can identify potential preventive strategies which can help in promoting the safety culture in healthcare organisations.

To conclude, the workflow delivering a clinical service must specify the tasks and processes to be completed as well as the people who are responsible to accomplish them in a particular order. Understanding this workflow is very important in order to maximize the efficiency and to prevent errors. A clear definition of the workflow can help in identifying its critical parts and in improving the flow of information and work within the healthcare organization. The involved parties must be involved in the development process of the workflow and they should have access to the final workflow and its information. They must be trained so that safety becomes an essential part of their work.

\section{Case Study: Radiology Department Workflow}

This section describes the application of the approach to a workflow within a radiology department. Whilst this is only a single example, we believe that the approach is applicable to many other clinical workflows.

In our project we accomplished an empirical study in a radiology department in a hospital in Jordan. We applied our approach to build and analyse the workflow within the department. The following subsections describe the steps: 


\section{Requirements Generation}

Initially, we documented the workflow within the radiology department and identified its components and the interactions between them. Critical workflow components were also determined. These are the components which potentially experience faults and errors as well as failures that are likely to happen if the component is compromised by the error. Data is collected from and discussed with a healthcare team in the department regarding the occurrence of workflow errors and their prevention in the same scenario environment. The result was a documentation of the involved components, their failure behaviour, and the interactions between them.

The following describes part of the details which are required to analyse one failure scenario:

The workflow starts with the Hospital Information System (HIS). The data is stored in the Electronic Health Record (EHR) which is a subcomponent of the HIS. The clinician is responsible for sending the data to the Radiology Information System (RIS), which acts with the Picture Archiving and Communication System (PACS) as an individual departmental radiology system. The information to be entered includes the following: Patient name, Patient National Number (ID), Date of Birth (DoB), Age, Address, Patient medical Information, and Order Information. Later scheduling information can be added into the RIS by a radiologist or a nurse.

Next, the RIS output goes directly to the Modality Worklist (MWL) which acts as a database in which the orders are scheduled to be sent automatically to the image acquisition modality. This database is exposed to hardware and software errors. At the image acquisition modality the patient is supposed to have an examination that is specified in the order. The output of the image acquisition modality is the patient id, patient name and the image itself. This output is transferred automatically to the PACS.

When PACS receives this output information it archives them and sends them to the diagnostic workstation to be seen by the radiologist. The radiologist interprets the examination and produces a report as an output. This report is then passed to the clinician to make the diagnoses and to deliver the treatment.

\section{Workflow Modelling}

The following figure shows the model which we developed to represent the workflow within the department: 
Figure 2. Radiology Workflow

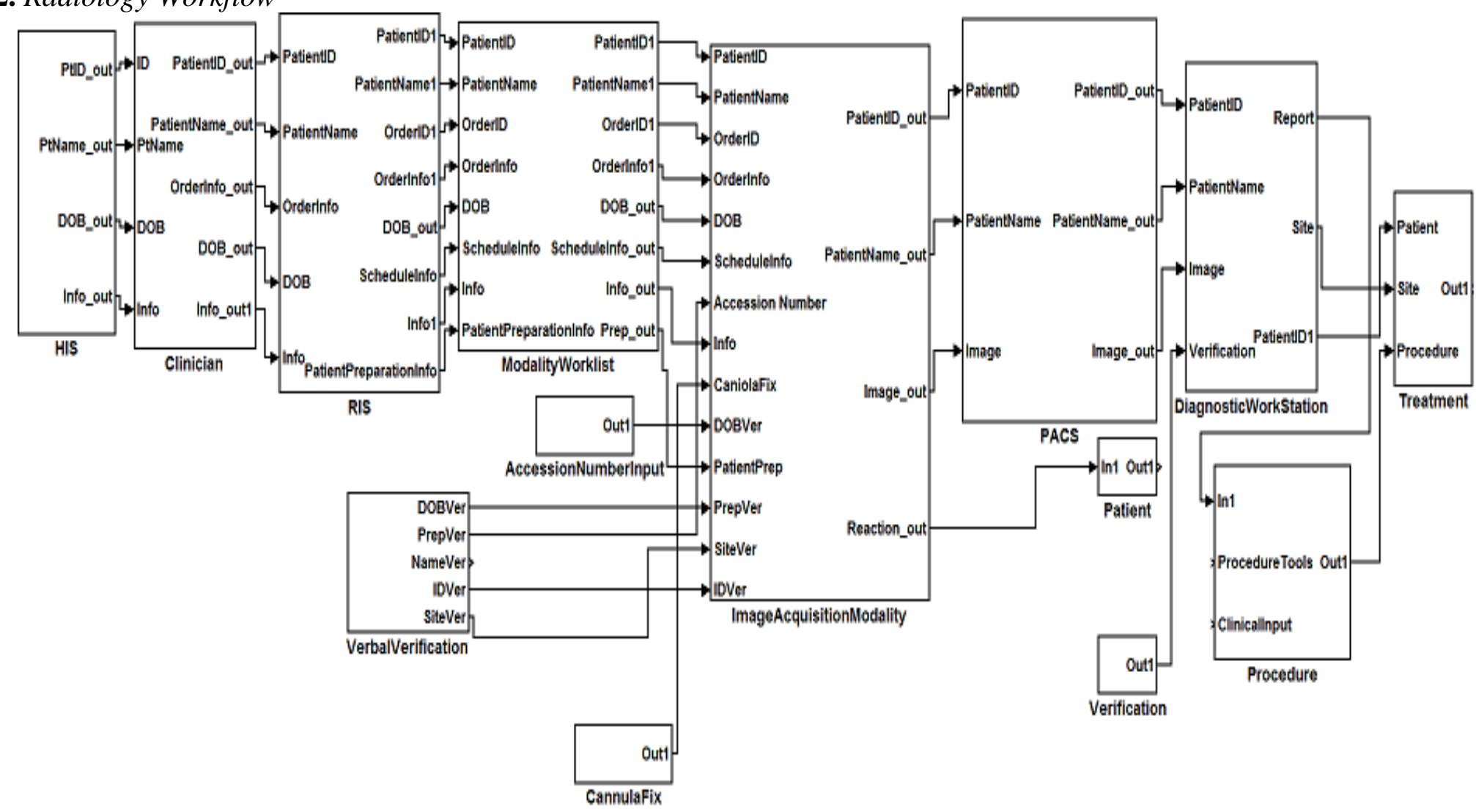


We built our model using Matlab Simulink which has an interface to HiPHOPS. The workflow components include health information systems, human agents, hardware components, tasks and processes.

\section{Hazards Identification}

The potential errors at component level were specified in cooperation with the healthcare team. Possible failures and internal malfunctions were discussed and identified.

\section{Failure Annotation}

Here, we extended the model with failure information. This information describes how a failure in the component output is caused by a propagation of failure from the component input or the internal malfunction of the component itself. Failure is represented in the format of "FailureType-ComponentName. ComponentPort" in HiP-HOPS.

We analysed the scenario on providing the wrong treatment to the patient. As described by the workflow architecture, the treatment is considered as a separate component, the value failure of the output is represented as V-Out1. For simplicity, in presenting our results we referred to the value failure of the output as "wrong treatment".

The patient's ID and DOB are entered into the HIS together with other information. Incorrect data entry may cause a value failure of ID and DoB which we denoted as V-ID_out, and V-DoB_out. Also omission of the DoB which is denoted as O-DoB causes problems and it is classified here as output deviation of the HIS. Moreover, HIS internal malfunctions can cause the output failures of the HIS; these are represented as HWError, SWError, and DataEntryError.

Similarly, the clinician - who is included in the workflow as a separate component - can have output deviations. The clinician might make data entry errors which are represented here as IDDataEntryError or DoBDataEntryError. The output deviations are represented as V-PatientID_out and V-DoB_out.

RIS internal malfunctions may include software or hardware malfunction, represented as HWError, SWError. RIS as well may receive wrong ID and wrong DoB from the Clinician, represented as IDDataEntryError and DoBDataEntryError. Therefore, output deviations at RIS could be having the wrong ID, omission of DoB, or having the wrong DoB; these are represented respectively as: V-ID_out, O-DoB_out, V-DoB_out.

MWL can have two basic events, which are software error or hardware error. These are represented as SWError and HWError respectively. Each of the ModalityWorklist inputs has its own failure but in the first scenario, some failures have been considered and the others are ignored as they are assumed to be free from failures. The failures which are to be analysed are: the failure of the value of the ID, and the value failure of the DoB either as a value failure or omission of this value. These are represented as V-ID_out, V-DoB_out.

When it comes to the image acquisition modality, at the time of the test the radiologist should verify certain information with the patient, e.g. ID, DoB, 
name, and the site of the image (e.g. right/left). The process of verbal verification is represented as a separate component which may have three basic events which are human errors; we represented them as: IDHumanError, DoBHumanError and SiteHumanError. Failures of the output of this component are represented as: O-IDVer, O-DOBVer and O-SiteVer. Basic events for the image acquisition modality are represented as ImageUnmarked, and ImageMislabled. These basic events happen when the image is mislabelled with the wrong patient or the wrong data, such as having the image for the right lung instead of the left one. The other case may happen when the image has the right information but it is unmarked for the purpose of specifying the site, and therefore the radiologist produce the wrong report.

The output deviations of the PACS component were represented as VPatient_out and V-Image_out. The diagnostic workstations component has the HumanError as a basic event, and has a number of output deviations: V-Report, V-Site, and V-PatientID.

The treatment procedure may have an output deviation as well, and this is represented as V-Out1, which might be caused by V-In1 OR ProcedureTool OR ClinicalInput.

Fault Tree and FMEA Synthesis and Analysis

The components of the model were annotated with the corresponding failure information and then we performed the root cause analysis. HiP-HOPS synthesises and analyses the system fault trees and produces the FTA and FMEA results, which show how the value failure in an input and the component failures (or their combinations) can lead to the failure in causing the wrong treatment of the patient.

The following figure shows the FTA:

Figure 3. FTA

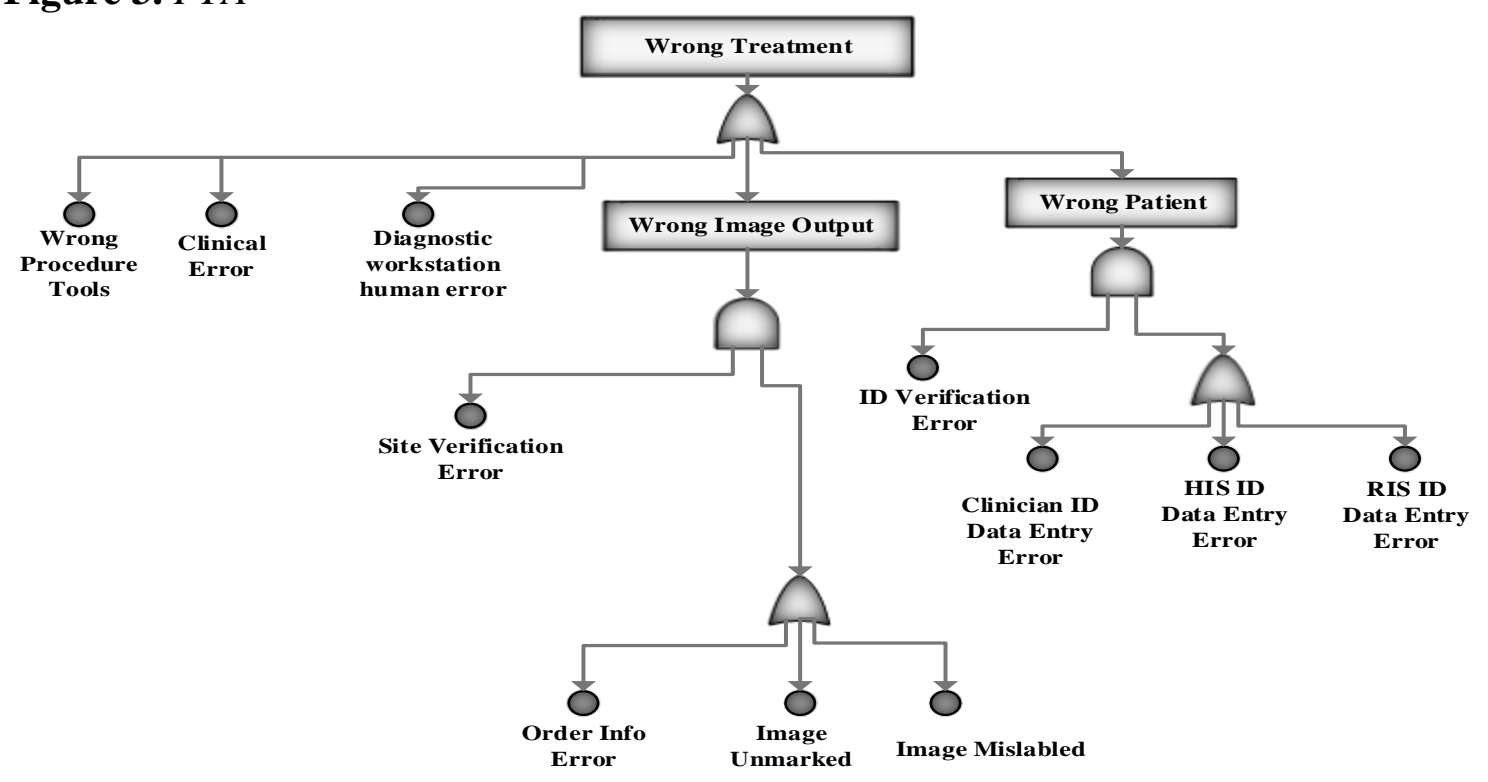


The following list shows the MCS from the FTA:

Figure 4. $M C S$

\begin{tabular}{|l||}
\hline Wrong Procedure Tools \\
\hline \hline Clinical Error \\
\hline Diagnostic Workstation Human Error \\
\hline \hline Site Verification Error $\underline{\text { AND }}$ Order Info Error \\
\hline \hline Site Verification Error $\underline{\text { AND } \text { Image Unmarked }}$ \\
\hline \hline Site Verification Error $\underline{\text { AND } \text { Image Mislabelled }}$ \\
\hline \hline ID Verification Error $\underline{\text { AND }}$ Clinician ID data entry \\
error \\
\hline \hline ID Verification Error $\underline{\text { AND }}$ HIS ID data entry error \\
\hline \hline ID Verification Error $\underline{\text { AND }}$ RIS ID data entry error \\
\hline \hline
\end{tabular}

The MCS is a smallest - i.e., necessary and sufficient - combination of primary events causing the top event. All of the basic events in the set need to occur to cause the top event. It directly links the top event to the primary events, and the complete set of the MCS provides the complete set of causes of the top event.

The following table shows the resulting FMEA table of the direct effects:

Table 1. FMEA of the Direct Effects

\begin{tabular}{|c|c|}
\hline \multicolumn{2}{|c|}{ Component: Diagnostic Workstation } \\
\hline Failure Mode & Workflow Effect \\
\hline Human Error & Wrong Treatment \\
\hline \multicolumn{2}{|c|}{ Component: Procedure } \\
\hline Failure Mode & Workflow Effect \\
\hline Wrong Procedure Tool & Wrong Treatment \\
\hline Wrong Clinical Input & Wrong Treatment \\
\hline
\end{tabular}


The following table shows the resulting FMEA table of the further effects:

Table 2. FMEA of Further Effects

\begin{tabular}{|c|c|}
\hline \multicolumn{2}{|c|}{ Component: Clinician } \\
\hline Failure Mode & Workflow Effect \\
\hline ID Data Entry Error & Wrong Treatment \\
\hline Order Info Error & Wrong Treatment \\
\hline Component: Image Acquisition Modality \\
\hline Failure Mode & Workflow Effect \\
\hline Image Unmarked & Wrong Treatment \\
\hline Image Mislabelled & Womponent: RIS \\
\hline Failure Mode & Workflow Effect \\
\hline ID Data Entry Error & Wrong Treatment \\
\hline \multicolumn{2}{|c|}{ Component: Verbal Verification } \\
\hline Failure Mode & Workflow Effect \\
\hline ID Verification Error & Wrong Treatment \\
\hline Site Verification Error & Wrong Treatment \\
\hline \multicolumn{2}{|c|}{ Component: HIS } \\
\hline Failure Mode & Workflow Effect \\
\hline ID Data Entry Error & Wrong Treatment \\
\hline
\end{tabular}

To summarise, the FTA and FMEA results show that the following faults may lead to the failure of the first scenario (which is in this case giving the wrong treatment to the patient):

- Human error at the diagnostic workstation, either by making an error in reading the image or the wrong specification of the site. The wrong specification of the site is usually a human error at the scheduling, at the order information level or a human error in the diagnostic workstation itself;

- The use of the inappropriate procedure tools;

- Incorrect clinical input for the procedure;

- Data entry error for the ID by a clinician, combined with an error in the verbal verification of the ID by the radiologist at the time of the imaging. This combination of errors may lead to the wrong treatment because of producing a report for the wrong patient;

- Data entry error for the ID in the RIS combined with an error in the verbal verification of the ID by the radiologist at the time of the imaging. Data entry error for the ID in the HIS combined with 
an error in the verbal verification of the ID by the radiologist at the time of imaging;

- Clinician order information error combined with the site verbal verification;

- Image unmarked at the image acquisition modality combined with site verification error;

- Image mislabelled with the wrong study combined with site verification error.

This means if there is any error in the data entry in HIS, clinician, and the RIS, combined with a situation where the radiologist does not verify (or verifies incorrectly) the data for ID or site information, the wrong treatment towards the patient will occur. We can prevent such errors through highlighting the critical tasks in the workflow and adding extra functionality, for example using bar coded patients and marking of sites.

\section{Probability Analysis}

As our qualitative analysis results did not show a need for a costly replacement of the existing components, probabilistic analysis was not necessary. So, we directly verified the workflow with the healthcare team to declare the formal workflow.

New Workflow Design and Accreditation of the Workflow

In our case the workflow which we built was sufficient as the healthcare team verified it and they were satisfied with the results. The approach suggests areas to improve those tasks which can cause failures. As the workflow will be available to the people involved in it, as part of building awareness, they should be made aware of the critical tasks and the potential failures they can cause so that they are able to accomplish them properly and safely.

\section{Conclusion}

It is concluded that the approach is applicable to this area of healthcare and provides benefits through a combination of detailed information on possible risks and descriptive safety analysis based on experts' opinion. This provides a mechanism for the systematic identification of both adverse events and possible safeguards in clinical workflows, which is important in terms of identifying the causes of possible adverse events before they happen and therefore helping to prevent harm to the patient. Moreover, the approach helps in the clear definition of the workflow including its processes and tasks, which provides a valuable opportunity for formulation of safety improvement strategies. 


\section{References}

Battles, J. \& Lilford, R. 2003. Organizing patient safety research to identify risks and hazards. Quality and Safety in Health Care, 12, ii2-ii7.

Belhajjame, K., Vargas-Solar, G. \& Collet, C. Year. A flexible workflow model for process-oriented applications. In: Web Information Systems Engineering, 2001. Proceedings of the Second International Conference on, 2001. IEEE, 72-80.

Fischer, L. 2001. Workflow handbook 2001. Lighthouse Point: Future Strategies.

Green, M. 2013. Error and Injury in Computers and Medical Devices. Available: http://www.visualexpert.com/Resources/compneg.html [Accessed 4/5/2013].

Murff, H. J., Patel, V. L., Hripcsak, G. \& Bates, D. W. 2003. Detecting adverse events for patient safety research: a review of current methodologies. Journal of Biomedical Informatics, 36, 131-143.

Ouvry, A. 2002. Workflow analysis and modeling in medical IT projects. Medicamundi, 46, 47-55.

Reason, J. 1990. Human error, Cambridge university press.

Ruffolo, M., Manna, M., Cozza, V. \& Ursino, R. Year. Semantic clinical process management. In: Computer-Based Medical Systems, 2007. CBMS'07. Twentieth IEEE International Symposium on, 2007. IEEE, 518-523.

Starfield, B. 2000. Medical Errors - A Leading Cause of Death. Journal of Medical Association (JAMA), 284.

Vesely, W. E., Goldberg, F. F., Roberts, N. H. \& Haasl, D. F. 1981. Fault tree handbook. DTIC Document. 